\title{
High Resolution Optical Remote Sensing Satellites - Challenges and Techniques
}

\author{
Palani Murugan, Gomathi Lakshmi, Vivek Kumar Gautam
}

\begin{abstract}
High spatial resolution satellite data is essential to identify small objects and extract minute details of the terrain. This data is provided by many satellites and being used in numerous applications. The realization of high resolution satellite is a challenging task. Significant complexity lies in the realization of high spatial resolution camera starting from material selection, high stiffness-low mass opto-mechanical system design, detector selection to high speed camera electronics design. The mass and size of camera increase with the improvement in spatial resolution. Alternate methods such as Step and Stare, and time delay integration methods can be used to achieve high signal to noise ratio images. The performance of satellite bus subsystems like structure, the data handling, and storage system, the data transmission system, attitude sensors and actuators should also be improved to achieve good quality data.

The data handling system has to be designed to handle high data rate and data volume. The capacity of the data storage system has to be increased to cater the high data volume storage requirement. The data transmission system needs to be sufficiently capable to transmit the high volume imaging data to the ground station. As the spatial resolution improves, the spacecraft pointing accuracy and drift rate requirements become stringent. Improved attitude sensors and high capacity actuators are essential to meet these stringent requirements. Generally, high resolution cameras are combined with high speed electronics to handle high data rates which need more power. In this paper, we discuss various techniques being employed to obtain high resolution data with reasonable SNR. The challenges involved and the improvements required in various spacecraft subsystems to support these high resolution cameras are presented. Techniques employed by different space agencies to obtain high spatial resolution images are also discussed. The characteristics of high resolution satellites are also tabulated and compared.
\end{abstract}

Key words: High resolution, Cartography, Remote Sensing, Spatial Resolution

\section{INTRODUCTION}

Satellite remote sensing data is being utilized in many fields as it provides wide area coverage in reduced time with fixed repeat cycle. The remote sensing data is generally

Revised Manuscript Received on June 05, 2020.

* Correspondence Author

Dr. Palani Murugan *, IRS Programme Management and Systems Group, U.R. Rao Satellite Centre, Bangalore, Karnataka India. E-mail: palanimuruga64@gmail.com

Gomathi Lakshmi, IRS Programme Management and Systems Group, U.R. Rao Satellite Centre, Bangalore, Karnataka India. E-mail: lgomathi@rediffmail.com

Vivek Kumar Gautam, IRS Programme Management and Systems Group, U.R. Rao Satellite Centre, Bangalore, Karnataka India. E-mail: vivekgiist@gmail.com

(C) The Authors. Published by Blue Eyes Intelligence Engineering and Sciences Publication (BEIESP). This is an open access article under the CC BY-NC-ND license (http://creativecommons.org/licenses/by-nc-nd/4.0/) specified by four different resolutions namely spatial, spectral, radiometric and temporal resolutions. These resolution requirements are derived based on applications. The resolution requirements for different applications are provided in table-1. Though all these resolutions are continuously improving, the progress of spatial resolution is commendable and its present value is around $0.30 \mathrm{~m}$ in panchromatic images at nadir view. The requirements of high resolution data call for improvements in camera system, spacecraft bus systems and data image processing chain. The high resolution satellites are being launched by many organisations /agencies for governmental and commercial applications

\begin{tabular}{|l|c|c|c|c|}
\hline \multicolumn{1}{|c}{ Table.I Applications Vs Resolutions } \\
\hline Application & $\begin{array}{c}\text { Spatial } \\
\text { Resolu } \\
\text { tion } \\
\mathbf{( m )}\end{array}$ & $\begin{array}{c}\text { Spectral } \\
\text { bands }\end{array}$ & $\begin{array}{c}\text { Radiometric } \\
\text { Resolution } \\
\text { (bits) }\end{array}$ & $\begin{array}{c}\text { Temporal } \\
\text { Resolutio } \\
\text { n } \\
\text { (Days) }\end{array}$ \\
\hline Agriculture & $5-50$ & $\begin{array}{c}\text { VIS-NIR } \\
\text { SWIR }\end{array}$ & 10 & 2 to 30 \\
\hline Forestry & $50-150$ & $\begin{array}{c}\text { VIS-NIR } \\
\text { SWIR- TIR }\end{array}$ & 8 & $\begin{array}{l}\text { Few } \\
\text { months }\end{array}$ \\
\hline $\begin{array}{l}\text { Limnology } \\
\text { Oceanography }\end{array}$ & $\begin{array}{c}20-100 \\
00\end{array}$ & VIS-NIR & $12-14$ & $\begin{array}{c}\text { Few } \\
\text { TIR }\end{array}$ \\
\hline $\begin{array}{l}\text { Disaster } \\
\text { management }\end{array}$ & $<10$ & VIS-NIR & 10 & $\begin{array}{l}\text { Few } \\
\text { hours }\end{array}$ \\
\hline Cartography & $<1$ & VIS & 12 & 2 -3 months \\
\hline
\end{tabular}

\section{Where VIS - Visible region \\ NIR - Near Infrared \\ SWIR - Shortwave Infrared \\ TIR - Thermal Infrared}

The satellite remote sensing for civilian applications started in 1972 with the launch of Earth Resource Technology Satellite (ERTS)which was later renamed as Landsat-1. This satellite was followed by many satellites with improved performance. The Landsat was followed by Satellite Pour l'Observation de la Terre(SPOT)series and Indian Remote Sensing Satellites (IRS). The first Satellite in SPOT series SPOT-1 and IRS-1A were launched in 1986 and 1988 respectively. These satellites were providing medium spatial resolution i.e. from 10 to $30 \mathrm{~m}$. Features of High resolution optical remote sensing satellite are compared in the Table-2. Data from these remote sensing satellites were extensively utilized in various applications like agriculture, forestry, oceanography, limnology, geology, glaciology, cartography etc. 
High Resolution Optical Remote Sensing Satellites - Challenges and Techniques

Table-II Specification of contemporary high resolution optical remote sensing satellites

\begin{tabular}{|c|c|c|c|c|c|c|c|c|c|c|c|c|c|}
\hline Sl.No & $\begin{array}{l}\text { Satellite } \\
\text { /Sensor }\end{array}$ & Country & $\begin{array}{l}\text { Date of } \\
\text { Launch }\end{array}$ & $\begin{array}{c}\text { Resolution } \\
\text { (m) }\end{array}$ & $\begin{array}{c}\text { Swath } \\
\text { (km) }\end{array}$ & $\begin{array}{c}\text { Altitude } \\
\text { (km) }\end{array}$ & $\begin{array}{c}\text { Focal } \\
\text { Length } \\
\text { (m) } \\
\end{array}$ & $\begin{array}{l}\text { Aperture } \\
\text { (cm) }\end{array}$ & Bits & $\begin{array}{c}\text { Revisit } \\
\text { (days) }\end{array}$ & $\begin{array}{c}\text { Mass } \\
(\mathbf{k g})\end{array}$ & $\begin{array}{c}\text { Power } \\
\text { (W) }\end{array}$ & $\begin{array}{l}\text { SSR } \\
\text { (Gb) }\end{array}$ \\
\hline 1 & IKONOS & USA & 24-09-1999 & 0.8 & 11 & 681 & 10 & 70 & 11 & 14 & 817 & 1500 & 64 \\
\hline 2 & EROS - A1 & Israel & $05-12-2000$ & 1.9 & 12.5 & 480 & 3.45 & 30 & 12 & $3-5$ & 280 & 450 & \\
\hline 3 & Quickbird-2 & USA & 18-10-2001 & 0.61 & 16.5 & 450 & 8.8 & 60 & 11 & & 1100 & 1500 & 128 \\
\hline 4 & SPOT 5 & France & 04-05-2002 & $2.5-5$ & 60 & 822 & 0.58 & 20 & 8 & 26 & 3085 & 2400 & 90 \\
\hline 5 & SPOT-6 & France & 09-09-2012 & 2 & 60 & - & - & - & - & 26 & 720 & & 850 \\
\hline 6 & Orbview3 & USA & 26-06-2003 & 1 & 8 & 470 & - & 45 & 11 & 3 & 304 & 625 & 32 \\
\hline 7 & Cartosat-1 & India & 05-05-2005 & 2.5 & 26 & 630 & 3.9 & 50 & 10 & 5 & 1,560 & 1100 & 120 \\
\hline 8 & ALOS & Japan & 24-01-2006 & 2.5 & 35 & 691 & 2 & 30 & 8 & 2 & 4000 & 7000 & 768 \\
\hline 9 & EROS-B1 & Israel & 25-04-2006 & 0.7 & 16 & 508 & 5 & 50 & 8 & $3-5$ & 350 & 800 & - \\
\hline 10 & Kompsat-2 & Korea & 28-07-2006 & 1 & 15 & 685 & 9 & 60 & 10 & - & 800 & 955 & 96 \\
\hline 11 & Worldview-1 & USA & 18-09-2007 & 0.5 & 17.6 & 494 & 8.8 & 60 & 11 & $1-4$ & 2500 & 3200 & 2199 \\
\hline 12 & Worldview-2 & USA & 08-10-2009 & 0.31 & 16.4 & 770 & 13.3 & 110 & 11 & $2-4$ & 2800 & 3200 & 2199 \\
\hline 13 & Worldview-3 & USA & 13-08-2014 & 0.31 & 13,1 & 617 & 13.3 & 110 & 11 & $1-4.4$ & 2800 & 3100 & 2199 \\
\hline 14 & Worldview-4 & USA & 11-11-2016 & 0.31 & 13.1 & 617 & 13.3 & 110 & 11 & 4.5 & 2600 & & 3200 \\
\hline 15 & Geoeye-1 & USA & 06-09-2008 & 0.41 & 15.2 & 681 & 13.3 & 110 & 11 & $4-8$ & 1955 & 3862 & 1000 \\
\hline 16 & Cartosat-2 & India & 10-01-2007 & 0,8 & 9.6 & 630 & 5.6 & 70 & 10 & 126 & 680 & 900 & 64 \\
\hline 17 & Cartosat-2A & India & 28-04-2008 & 0,8 & 9.6 & 630 & 5.6 & 70 & 10 & - & 690 & 900 & 64 \\
\hline 18 & Cartosat-2B & India & $12-07-2010$ & 0,8 & 9.6 & 630 & 5.6 & 70 & 10 & - & 694 & 900 & 64 \\
\hline 19 & Cartosat-2C & India & 22-06-2016 & 0.66 & 10 & 505 & 5.6 & 70 & 11 & - & 727 & 900 & 64 \\
\hline 20 & Cartosat-2D & India & $15-02-2017$ & 0.66 & 10 & 505 & 5.6 & 70 & 11 & - & 714 & 986 & 64 \\
\hline 21 & Cartosat-2E & India & 23-06-2017 & 0.66 & 10 & 505 & 5.6 & 70 & 11 & - & 712 & 986 & 64 \\
\hline 22 & Cartosat-2F & India & 12-01-2018 & 0.66 & 10 & 505 & 5.6 & 70 & 11 & - & 680 & 986 & 64 \\
\hline 23 & Cartosat-3 & India & 27-11-2019 & 0,3 & 15 & 509 & 15 & 120 & 11 & - & 1625 & 2000 & 1000 \\
\hline 24 & Kompsat-3 & Korea & 17-05-2012 & 0.7 & 15 & 685 & 8.6 & 80 & 14 & 3 & 980 & 1300 & 512 \\
\hline 25 & Kompsat-3A & Korea & 25-5-2015 & 0.55 & 12 & 528 & 8.6 & 80 & 14 & 3 & 1100 & 1300 & 512 \\
\hline 26 & Pleiades-1A & France & $17-12-2011$ & $0.5-2$ & 20 & 695 & 12.9 & 65 & 12 & $1-3$ & 970 & 1500 & 600 \\
\hline 27 & Pleiades-1B & France & 02-12-2012 & $0.5-2$ & 20 & 695 & 12.9 & 65 & 12 & $1-3$ & 970 & 1500 & 600 \\
\hline 28 & Skysat-1... & USA & 21-11-2013 & 0.8 & 8 & 600 & 3.6 & 35 & 11 & - & 84 & 120 & 768 \\
\hline 29 & Gaofen-1 & China & 26-04-2013 & 2 & 60 & 645 & - & - & 10 & 4 & 1080 & 1278 & 3700 \\
\hline 30 & Gaofen-2 & China & 19-08-2014 & 0,8 & 45 & 631 & 7.8 & 50 & 10 & 4 & 2100 & 3200 & 3756 \\
\hline
\end{tabular}

Around five-meter resolution data became available from IRS-1C/1D satellites at the end of last century. These data were used in cartography, infrastructure planning and strategic applications.

The IKONOS satellite, launched on $24^{\text {th }}$ September, 1999 by Digital globe was the first commercial satellite to provide sub-meter spatial resolution data. This was followed by many satellites like Worldview, Geoeye, Quickbird, Kompsat, Cartosat-2 etc.

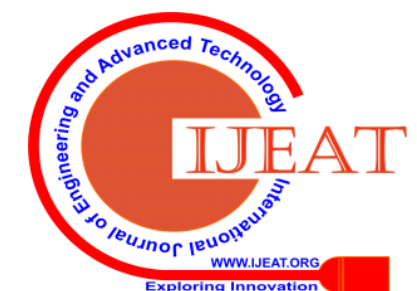


These data are used for urban and rural mapping [1,2], disaster damage estimation [3], agriculture and forestry studies, mining, infrastructure planning, seismic risk assessment[4-6] and change detection. Small satellite constellations are also used to obtain high resolution remote sensing data [7].

\section{SPATIAL RESOLUTION}

Spatial resolution can be defined as the projection of the pixel on ground [8]. In simple words, the spatial resolution is the size of the smallest object that can be resolved by the sensor. In satellite remote sensing, the spatial resolution can be divided in two parts namely across track resolution and along track resolution.

The along track resolution is sampling distance in the direction of satellite movement, dictated by the ground trace velocity and the integration time. The relation between them is provided in equation (1).

$$
\mathrm{R}_{\mathrm{l}}=\mathrm{T}_{\mathrm{i}} \times \mathrm{GTV} \quad-(1)
$$

Where, $\mathrm{R}_{\mathrm{l}}$ is along track resolution

$$
\begin{aligned}
& \text { Ti - Integration Time } \\
& \text { GTV - Ground Trace Velocity }
\end{aligned}
$$

The Ground Trace Velocity is based on the radius of the Orbit

$$
\mathrm{GTV}=\mathrm{V}_{\mathrm{s}} \mathrm{x} R /(\mathrm{R}+\mathrm{h}) \quad-(2)
$$

Where, $\mathrm{V}_{\mathrm{s}}$ - Satellite orbital velocity

$$
\begin{aligned}
& \mathrm{R} \text { - Radius of Earth } \\
& \mathrm{h} \text {-Altitude }
\end{aligned}
$$

The satellite velocity is related to the radius of the orbit and can be given as

$$
\mathrm{Vs}=(\mathrm{GM} / \mathrm{R})^{1 / 2}
$$

Where, G - Gravitational constant

$$
\begin{aligned}
& \mathrm{M} \text { - is mass of Earth } \\
& \mathrm{R} \text { - is radius of the orbit }
\end{aligned}
$$

Generally, the shape of the pixels in the detectors is square. If the pixel is rectangle, the integration time is adjusted to get square pixels in the image.

The across track resolution is associated with the focal length of the optical system, pixel size of detector and altitude of satellite. The relation between them is provided in equation (4).

$$
\mathrm{R}_{\mathrm{c}}=\mathrm{d} \times \mathrm{h} / \mathrm{f}
$$

Where, $\mathrm{R}_{\mathrm{c}}=$ Across track spatial resolution

$\mathrm{d}=$ Pixel size of the detector.

$\mathrm{h}=$ Altitudeof the satellite

$\mathrm{f}=$ Focal length of optical system.

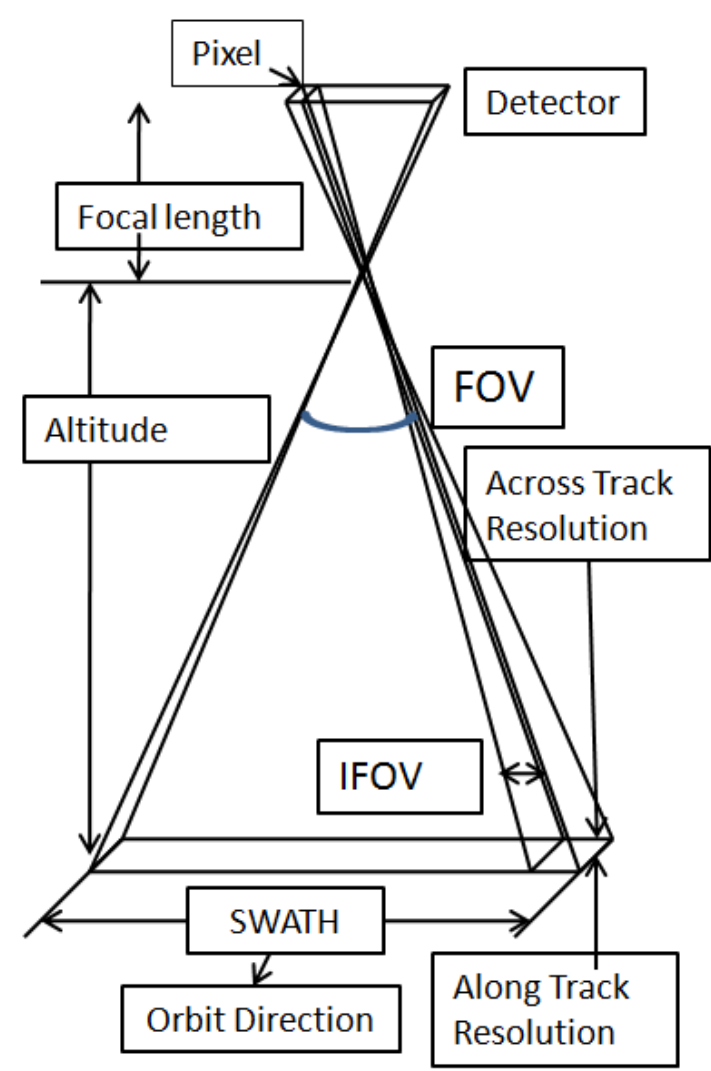

Fig. 1: Imaging configuration

The resolution can be improved by any one or combination of the following four options.

1. Reducing the size of detector pixel,

2. Lowering the satellite altitude

3. Increasing the focal length and

4. Using Super Resolution detectors

When spatial resolution improves, number of pixels required to cover specified swath increases. As accommodating more pixels in single detector is technically difficult, higher number of detectors are used for covering full swath.

\section{A. Reducing the pixel size}

Reducing the pixel size improves the spatial resolution linearly. Reduction of the pixel size is based on the semiconductor technology development. At present the smallest pixel available in linear array Charge Coupled Device (CCD) is around $7 \times 7$ microns. The main disadvantage is that reduction in the pixel size leads to reduction in the signal collected by the pixel and signal to noise ratio(SNR).

\section{B. Lowering the satellite altitude}

Lowering the satellite altitude improves the spatial resolution. But it affects other parameters of the products. The implications due to the altitude reduction are as follows.

1. Decreasing the altitude below $500 \mathrm{~km}$ leads to increase in atmospheric drag which may reduce the lifetime of the mission or call for more fuel.

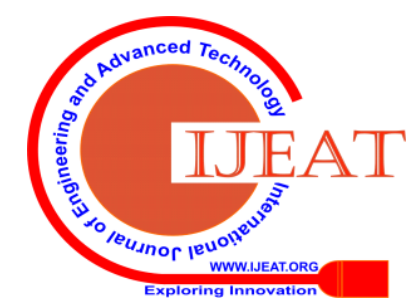




\section{High Resolution Optical Remote Sensing Satellites - Challenges and Techniques}

2. Atomic oxygen effect on the solar panels and spacecraft surface.

3. Total coverage area with nadir views reduces.

4. Lowering altitude reduces the swath compared to higher altitudes.

5. If satellite is in a constellation, more satellites need to be added to get the same coverage area.

\section{Increasing focal length}

Increasing the focal length is the preferred option to improve the spatial resolution. But this option increases the optical system overall size and mass. In detector based imaging systems, there are two types of angular resolutions. They are diffraction limited resolution and detector size limited resolution [9].

The diffraction limited resolution is angular resolution of optical system provided by Rayleigh's criterion. This is generally accepted criterion which states that two point sources are resolvable if first diffraction minimum of the image of one point source coincides with the maximum of another. The resolving power of an optical system can be provided as

$$
\theta_{0}=1.22 \lambda / \mathrm{d} \quad-(5)
$$

where, $\theta_{0}$ is in radians,

$\lambda$ is the wavelength in meters, and

$\mathrm{d}$ is the diameter of telescope in meters.

The detector limited angular resolution of the camera is given by

$$
\theta_{\mathrm{d}}=\mathrm{df}
$$

Where $\mathrm{d}=$ detector size and

$$
\mathrm{f}=\text { focal length. }
$$

However, one should ensure that the diffraction limited resolution of the optical $\operatorname{system}\left(\theta_{0}\right)$ is better(smaller) than detector limited resolution $\left(\theta_{\mathrm{d}}\right)$ to get better image quality of the products.

\section{SIGNAL STRENGTH}

Increasing the focal length reduces the signal available to the detector at the focal plane. The image size at the focal plane for a fixed field of view is called as format size. It increases with increase in focal length. The relation between them is provided in following equation.

$$
\begin{aligned}
& \text { The format size }=\mathrm{f} \times \theta . \\
& \begin{array}{l}
\text { Where, } \mathrm{f}=\text { focal length and } \\
\theta=\text { field of view }
\end{array}
\end{aligned}
$$

As the signal collected by the optical system is distributed to more area, the signal per unit area decreases. The same signal level can be retained by having same f- number. The Fnumber of an optical system is ratio of the focal length to aperture size and provided in equation (8).

$$
\text { The f-number }=f / D
$$

Where $\mathrm{f}=$ focal length

$$
\mathrm{D}=\text { Aperture size. }
$$

The f-number is also called as the speed of the lens.
Higher f-number corresponds to longer focal length which reduces signal availability to the detector. The f-number may be kept constant by increasing the aperture size along with the focal length. High aperture size systems give more signals to the detectors. The difficulties in realizing high aperture optical systems with lens system are

1. Availability of high volume glass material with uniform refractive index without stress and air bubble.

2. Mounting the lens is possible with peripheral (edge) contact only. This calls for considerable edge thickness.

3. Increasing the aperture size increases, the volume and mass. The increase in mass and the possibility of mounting the lens at edge only lead to the stress due to the self-weight.

4. As the optical ray travels in same direction in lens system, the overall length of optical system increases.

Therefore, high resolution imaging systems are realized with reflective optical elements [10] and its advantages are as follows.

1. As reflection is surface phenomenon, uniform material quality (refractive index, stress) is not required.

2. The chromatic aberration is zero.

3. Mirror can be held at many locations of back side and edges. This reduces the stress on the mirror due to self weight.

4. The mass of mirror can be reduced by scooping the back side of mirror (upto $90 \%$ mass reduction can be achieved).

5. As the optical rays are folded at the optical surface, the overall length of optical system comes down.

During the reflective optical system design following disadvantages are also to be considered.

1. Alignment of reflective system is critical compared to refractive system. Misalignment of reflective surface by $\theta$ degree may lead to deviation of optical ray by $2 \theta$ degrees, whereas it is $\sim \theta / 2$ in the case of refractive surface.

2. High reflective optical coating is required to retain high signal level

The total amount of signal collected by the detector is

$$
\mathrm{S}=\mathrm{s} \times \mathrm{t}
$$

where $\mathrm{s}$ - signal collected by detector per unit time and $\mathrm{t}$ - Integration time.

In the case of high resolution imaging system, the signal available at image plane decreases due to following reasons.

1. If the spatial resolution is improved by increasing the focal length, the format size increases and the energy will be distributed on wider area. Due to this, the signal collected by detector comes down. The signal collected by the detector per unit time is directly proportional to the $\mathrm{D}^{2}$ where $\mathrm{D}$ is the resolution.

2. The integration time also comes down due to the high resolution. The integration time $t$ is directly proportional to the Resolution D. 
Thus, improving the resolution, reduces the signal availability to the detector and the Signal to Noise Ratio (SNR).

High resolution satellites employ different techniques to improve the SNR. Important among them are

1. Increasing the aperture of the Optical system.

2. Step and stare imaging method

3. Using Time Delay Integration (TDI) detectors and

4. Adopting Super resolution methods

5. Using multi aperture system

\section{A. Increasing the Aperture Size}

If the focal length of the optical system increases to get high resolution, the signal received by the detector will decrease. The reduction in the signal can be compensated by increasing the aperture size. However, increasing the aperture beyond a certain limit may not be possible due to the constraints from mirror fabrication \& testing facilities, mirror mass and launch vehicle heat shield size.

\section{B. Step and stare method of imaging.}

The amount of the signal collected by the detector is signal available at image plane multiplied by integration time. In normal payload operation, the camera view direction velocity is equal to the ground trace velocity of the satellite.

To collect more signals at the detector, the camera view direction velocity is slowed down and the dwell time is increased. This increase in dwell time increases signal collected by the detector. The figure- 2 shows the 'Step and Stare' operation.

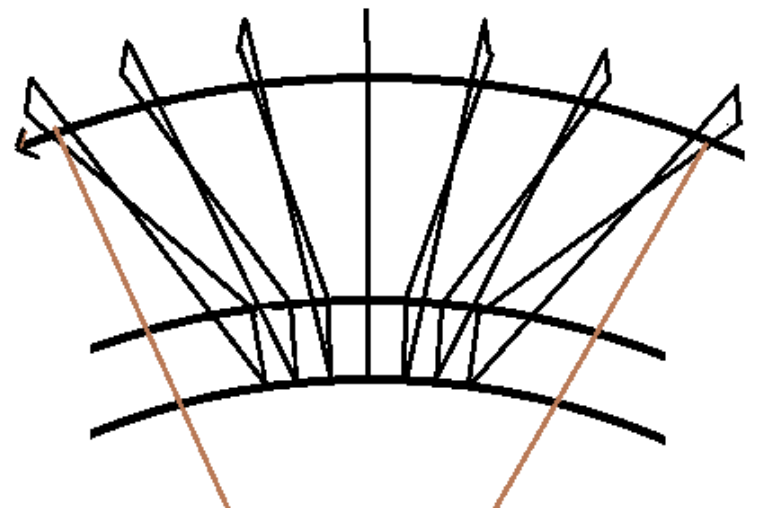

Fig. 2: Step and Stare Payload operation

\section{Time Delay Integration(TDI) detectors.}

In TDI detectors, many linear detectors are arranged parallel to each other to get an area array detector perpendicular to the satellite movement direction. At the end of integration time, the signal collected by one pixel in first row of detector is transferred to the same pixel in next row. The new pixel collects signal from the target and add to the signal received from previous row. At the end of integration time, this added signal is transferred to next row. This operation continues up to the last row. The figure- 3 shows the TDI imaging
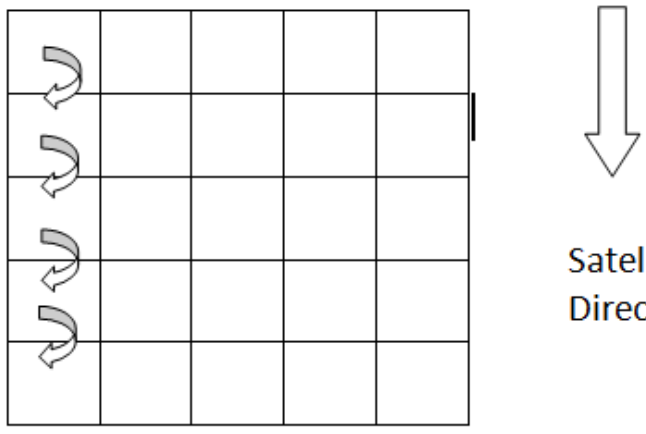

Satellite

Direction

Fig. 3: Time Delay Integration Detector (TDI)

In this method, the signal is multiplied by number of linear arrays arranged (stages) in the TDI detector and thus improves the SNR.

\section{Super resolution detectors}

Super resolution is a technique of obtaining a high resolution image from low resolution images $[11,12]$. The images may be obtained from two different satellites or from same satellite but from two different dates. If two images are obtained simultaneously, the illumination and look angle conditions will be same. This will reduce the difficult image preprocessing activities. This type of images can be obtained by mounting two detectors at the image plane in staggered manner as shown in figure-4

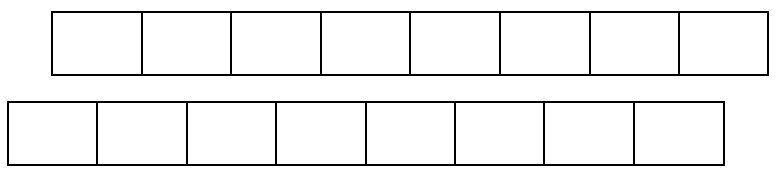

Fig. 4: Staggered detector arrays for Super resolution

High resolution image is constructed from the low resolution images obtained from both detectors. In this method, the focal length requirement is shorter. Correspondingly the size, mass of the camera system also comes down.

\section{E. Multi aperture system}

In the case of multi aperture system, multiple imaging systems with high F-numbers are aligned to view same location. Virtually the $\mathrm{F}$ number is reduced by adding multiple small apertures. The signal collected by all imaging systems is added together to get better Signal to Noise Ratio [13]. In this method, large aperture mirror can be avoided but number elements and systems increases.

\section{SATELLITE BUS ELEMENTS}

\section{A. Structure}

The high resolution remote sensing cameras are sensitive to the thermal effects and the micro-vibrations. The selection of mechanical structure material and the design is an important consideration in high resolution satellite realization.

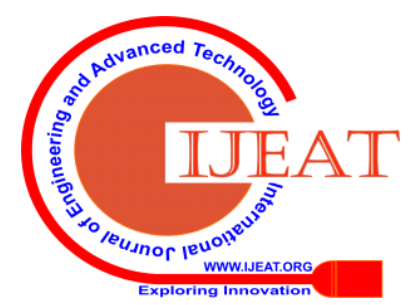




\section{High Resolution Optical Remote Sensing Satellites - Challenges and Techniques}

The structure should be stiff enough and thermally insensitive to maintain the look angles of imaging camera and the attitude sensors in intended directions [14]. The thermal expansion of the material and the micro-vibrations due to moving elements affect the image quality considerably. To combat the effect due to temperature variation, low thermal expansion coefficient materials are used in opto-mechanical systems. The micro-vibrations due to moving elements like Gyro, reaction wheels are suppressed by mounting them on vibration damping materials or systems $[15,16]$.

\section{B. Thermal Control System}

Generally, the satellite thermal system is designed with passive thermal elements like Multi-Layer Insulators (MLI), Optical Solar Reflectors (OSR), thermal paints, thermal tapes and copper brides/plates. High resolution satellite systems consume more power they dissipate more thermal energy. However, the temperatures of the systems are to be within a stringent temperature limit to get improved performance. This is achieved with mass heat transfer elements like heat pipes, embedded heat pipes and active thermal control elements like heaters and coolers [17-19].

\section{Data handling system}

The data handling system collects data from the payload(s), formats it suitably and delivers to the data transmission system. In addition to this, based on the requirement from the user, the error correction codes and encryption are incorporated by data handling system. The mass of the data handling system is reduced by implementing these functions in software and modular hardware approach [20]. In high resolution remote sensing satellites, the data volume and data rate are high. The design of high speed electronics is such that it can handle the high data rate is challenging.

\section{Data compression system}

In high resolution satellite, due to more samples, the data rate is high. This data can be transmitted to ground station with narrow bandwidth after compression. The data is compressed using either lossless or lossy compression methods [21]. While the compression ratio of lossless compression method is less, the lossy compression techniques provide high compression ratio, but compression ratio has to be finalized with the acceptance of the user as it should not affect the applications. The satellites employ different compressions methods such as Differential Pulse Code Modulation (DPCM), Joint Photographic Experts Group (JPEG-Baseline), Consultative Committee for Space Data Systems-Lossless data compression (CCSDS-LDC), Consultative Committee for Space Data Systems-Image Data compression (CCSDS-IDC) andJ-PEG2000 [22,23]. They are implemented in Field Programmable Gate Arrays (FPGA) or Application Specific Integrated Circuits(ASIC).

\section{E. Data storage system}

If satellite collects data when it is not visible to the data reception system, the data is to be stored and delivered during ground station visibility period. The capacity of the data storage system is arrived at based on the data rate and recording time per orbit and number of orbits not visible to the ground station. Also, when the output of the compressed data rate is more than the capacity of the transmission system, the excess data will be stored in the storage system. The stored excess data will be transmitted during non-imaging time of satellite in the night orbits.

\section{F. Data transmission system}

The data transmission system collects data from the data handling system, modulates the data and transmits to the ground station. Various modulation techniques like BPSK, QPSK and 8PSK are used and the data is transmitted in S-Band, X-band and Ka band to ground stations. The high resolution satellites are designed to operate in off-nadir modes to cover important locations. The process of Data collection in off-nadir mode and the data transmission to the ground station are to be carried out simultaneously. This is possible by electronic steering of the microwave beam towards the ground station using the Phased Array Antenna (PAA) or by using mechanically steerable antenna in spite of the look direction of imaging camera. Modulating high data rate and transmitting them to ground station are the challenges in realizing high data rate transmission systems. As water drops attenuate the Ka band transmission, it cannot be used during rainfall.

\section{G. Attitude and orbit control system}

The attitude and orbit control system (AOCS)consists of attitude sensors, orbit determination system and actuators. In High resolution satellites all these systems are to be improved.

\section{1) Attitude Sensors}

Attitude Sensors provide the attitude information (orientation of the satellite in the space) of the satellite. The high resolution remote sensing data needs accurate attitude knowledge for spacecraft maneuvering and data processing [24]. Accurate attitude information is obtained from high accuracy star sensors [25, 26]. Multiple head star sensor provides improved performance [27, 28]. Maintaining constant angle between these heads is important. If the Earth or moons come in field of view of the star sensor, sensor may not provide reliable attitude information. Under this condition, inertial systems like fiber optic gyros are used to provide high accuracy attitude information.

\section{2) Orbit Sensors}

Orbit sensors provide the information of location of satellite in the orbit. These sensors estimate the location of the satellite from the location of navigation satellites and the time of flight of microwave radiation from this navigation satellite. These satellite positioning systems provide sub meter location accuracy. Different Navigation satellites used are, Global Positioning System (GPS), Galileo, Globalnaya Navigazionnaya Sputnikovaya Sistema, or Global Navigation Satellite System(GLONASS), Indian Regional Navigation Satellite System (IRNSS), Beidou etc.

\section{3) Actuators}

Actuators are the devices used to rotate or increase the velocity of the satellites. In the spot imaging modes, the spacecraft has to be maneuvered faster to point the camera view from one imaging location to another location. This agility is achieved either by using high 
power Reaction Wheels(RW)[29] or Control Moment Gyros(CMG)[30, 31]. Currently, CMGs are used for agile high resolution remote sensing satellites.

\section{H. Power system}

Power system consists of Solar Array to generate power from sun light, Battery for storing the energy for the eclipse duration and DC/DC converters to generate required voltages for different subsystems. The high data rate camera, data handling system, data transmission system, Active thermal control system and high capacity actuators need more power, but at the same time the spot mode operation needs highly agile satellite for faster maneuver. Designing a power system to meet these contradicting requirements is essential and challenging. These contradicting requirements are met using Carbon Fiber Reinforced Plastic (CFRP) solar panels with high efficiency multi junction solar cells [32]. Payload operations in multiple orbits and data transmission in night orbit pass need high capacity battery. High efficiency batteries are designed with $\mathrm{Li}+$ ion cells.

\section{CONCLUSION}

Various challenges in realization of high resolution Imaging system are discussed. Methods to improve the spatial resolution were presented. Various methods to get higher Signal to Noise Ratio are provided. The improvement required in the satellites subsystems to support the high resolution payload is also discussed. Comparison of different high resolution satellites are made and presented in a table.

\section{ACKNOWLEDGEMENT}

Authors thank Shri C.A. Prabhakar, Ex. Project Director, Cartosat-3 and Shri G.Nagesh, Ex. Programme Director for their guidance. They also wish to thank Shri PJVKS Prakasha Rao, Programme Director SI: LEO \& PP for his support and Shri P.Kunhikrishnan, Director, UR Rao Satellite Centre, Bangalore for his continuous encouragement.

\section{REFERENCES}

1. Puissant. A. and Weber. C.(2002). The utility of very high resolution images to identify urban objects. Geocarto International, 17, 33-44.

2. Rahman Momeni, Paul Aplin, and Doreen S. Boyd, (2016). Mapping Complex Urban Land Cover fromSpaceborne Imagery: The Influence of Spatial Resolution, Spectral Band Set and Classification Approach, Remote Sensing, 8, 88.

3. Stefan Voigt, Thomas Kemper, Torsten Riedlinger, Ralph Kiefl, Klaas Scholte, and Harald Meh, (2007). Satellite Image Analysis for Disaster and Crisis-Management Support, IEEE Transactions on Geoscience and Remote Sensing, Vol. 45, No. 6, pp. 1520-28.

4. Zhongxia Yuan and Lanmin Wam, (2004). Application of highresolution satellite image for seismic risk assessment. 13th World Conference on Earthquake Engineering Vancouver, B.C., Canada August 1-6.

5. Maglione.P, (2016). Very High Resolution Optical Satellites: An Overview of the Most Commonly used, American Journal of Applied Sciences, Vol.3, No.1, 91-99.

6. Robert Ryan, Braxton Baldridge, Robert A. Schowengerdt, Taeyoung Choi, Dennis L. Helder and Slawomir Blonski, (2003). IKONOS spatial resolution and image interpretability characterization, Remote Sensing of Environment, 88, 37-52.

7. Kiran Murthy, Michael Shearn, Byron D. Smiley, Alexandra H. Chau, Josh Levine and M. Dirk Robinson,(2014). SkySat-1: very high-resolution imagery from a small satellite, Proceedings Volume
9241, Sensors, Systems, and Next-Generation Satellites XVIII; 92411E

8. Peighani-As.E, Abbasi-Moghadam. D, Ghafary. B and Tabataba-Vakili.V,(2009). Electro-Optical Design of Imaging Payload for a Remote Sensing Satellite, Journal Space science and Technology, Vol. 2, No. 5, 1-14.

9. Don Light, "A Basis for Estimating Digital Camera Parameters", Photogrammetric Engineering \& Remote Sensing Vol. 70, No. 3, pp. 297-300, 2004.

10. Ching-Wei Chen and Chia-Ray Che,(2019). Comprehensive Design and Analysis of a RC-Cassegrain Telescope for Micro-Satellite Mission, International Journal of Nano Research, Vol.2, No.1.

11. Lin Li, WeiWang, Heng Luo and Shen Ying, (2017). Super-Resolution Reconstruction of High-Resolution Satellite ZY-3 TLC Images, Sensors, 17, 1062.

12. R. Reulke, U. Tempelmann, D. Stallmann, M. Cramera and N. Haalaa, "Improvement of spatial resolution with staggered arrays as used in the airborne optical sensor", ADS40Commission I, WG I/4.

13. Bo Zhang, Keiichiro Kagawa, Taishi Takasawa, Min WoongSeo, Keita Yasutomi and Shoji Kawahito, (2014). RTS Noise and Dark Current White Defects Reduction Using Selective Averaging Based on a Multi-Aperture System, Sensors 14, 1528-154.

14. Kun Zhang, Xing Zhong, Guo Zhang, Deren Li, ZhiqiangSu, Yao Meng and Yonghua Jiang, (2019). Thermal Stability Optimization of the Luojia 1-01 Nighttime Light Remote-Sensing Camera's Principal Distance, Sensors, Vol.19, No.5, 990.

15. Lin Li, Luyang Tan, Lin Kong, Hongbo Yang and Dong Wang,(2017). Flywheel micro-vibration characters of high resolution optical satellite, VOL. 19, No.6 pp. 3981-3993.

16. Li Weipenga, Huang Haia Zhou Xubinb, Zheng Xintaoa and Bai Yang, (2014). Design and experiments of an active isolator for satellite micro-vibration, Chinese journal of aeronautics, Vol.27, No.6 pp. 1461-1468.

17. Abhijit,A,Adoni, Amrit Ambirajan, V.S.Jasvanth and D.Kumar,(2007). Thermohydraulic Modeling of Capillary Pumped Loop and loop heat Pipe, Journal of Thermo-physics and Heat Transfer, Vol.21, No.2.

18. K. N. Shukla, (2015) "Heat Pipe for Aerospace Applications-An Overview", Journal of Electronics Cooling and Thermal Control, 5, 1 14.

19. V.S. Jasvanth, Abhijit A. Adoni,V. Jaikumar and Amrit Ambirajan, (2016). Design and testing of an ammonia loop heat pipe, Applied Thermal Engineering 111.

20. Lalitkrushna Thakar, Chayan Dutta, P S Sura and S Udupa, (2016) Design \& realization of multi mission data handling system for remote sensing satellite,International Conference on Advances in Computing, Communications and Informatics (ICACCI).

21. B.Purna Kumari, (2018) "A Lossy Source Coding Algorithm Using The Least Significant Bits", International Journal of Scientific \& Engineering Research Vol.9, No.9, pp.1264-89.

22. Guoxia Yu, Tanya Vladimirova and Martin N. Sweeting, (2009). Image compression systems on board satellites, Acta Astronautica 64, pp. 988-1005.

23. P.S.Jagadeesh Kumar, J.Nedumaan, J.Tisa and J.Lepika, (2015) “A Comparative Case Study on Compression Algorithm for Remote Sensing Images, WCECS San Francisco, USA, October, 21-23, Vol 25, No.12, pp. 25-29.

24. Ganeshan.M, Padmasree.S and G. Nagendra Rao, (2007). Autonomous Star tracker for Agile Spacecraft, Journal of Spececraft Technology, Vol.17, No.1, pp. 20-25.

25. Muralidhara.K.R, Ashrita.M.S , Arpana.A. Padmasree.S, Srinivas Rao.J, Shashikala.T.H and Nagendra Rao.G, (2013). High speed autonomous embedded software for high accuracy star sensors, 2013 International Conference on Advanced Electronic Systems (ICAES), held at Pilani during 22-23 September.

26. Zhang Hao, Yanxiong Niu, Jiazhen Lu, and He Zhang, (2017). Accurate and Autonomous Star Acquisition Method for Star Sensor under Complex Conditions, Mathematical Problems in Engineering, Vol. 2017, Article ID 1643967.

27. Ho.K. and Nakasuka.S.,(2010). Novel star identification method combining two star trackers with extended FOVs, .In Proceedings of the AIAA Guidance, Navigation, and Control Conference, Toronto, ON, Canada,2-5 August 2010; pp. 8448.

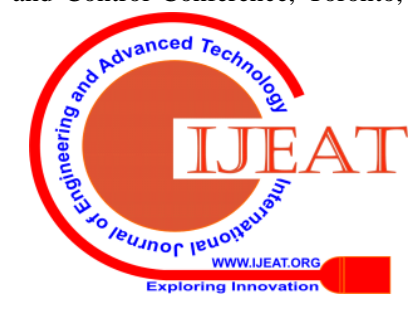


28. Majewski.L, Blarre.L, Perrimon.N, Kocher.Y, Martinez. P.E and Dussy.S, (2017). Hydra multiple head star sensor and its in-flight self-calibration of optical heads alignment" Proceedings Volume 10566, International Conference on Space Optics - ICSO 2008; 105660T.

29. Erna Kusuma Wati, Yulio Ramadhan, Hari Hadi S (2020). Control of 3-Axis Satellite Reaction Wheel using PID Control Optimized GeneticAlgorithms, nternational Journal of Engineering and Advanced Technology (IJEAT),Vol.9, No.3. February, 2020.

30. Jasim Ahmed, and Dennis S. Bernstein, (2002). Adaptive Control of Double-Gimbal Control-Moment Gyro with Unbalanced Rotor, Journal of Guidance control, and dynamics, Vol. 25, No. 1.

31. Abilash Mony, Hari Hablani and Gireesh N Sharma,(2016). Control Moment Gyro (CMG) Sizing and Cluster Configuration Selection for Agile Spacecraft, 10th National Symposium and Exhibition on Aerospace and Related Mechanisms (ARMS 2016) held at Thiruvananthapuram, Kerala, India, November 18-19.

32. T. V. Torchynska and G. Polupan, (2004) High efficiency solar cells for space applications, Superficies y Vacío Vol.17, No.3, pp.21-25.

\section{AUTHORS PROFILE}

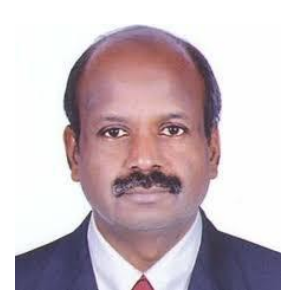

Dr. Palani Murugan obtained M.Sc, M.Tech and MBA degrees from Annamalai University, Anna University and Madurai Kamaraj University respectively. He received Ph.D from SRM University Chennai. He joined ISRO in 1990, after a short stay in Indian Institute of Science. He has worked for more than 20 Remote Sensing satellites in various capacities. At present he is heading Indian Remote Sensing Satellite (IRS) Programme Management \& Systems Group(PMSG), UR Rao Satellite Centre. He has conducted many training courses on spacecraft and their applications. His areas of interest include Project Management, Electro-optical Systems, Remote Sensing, Environment, Water Quality and Digital Image Processing.

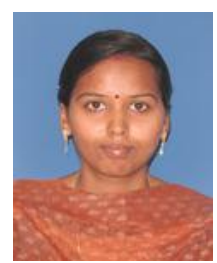

Gomathi Lakshmi obtained B.E. degree in Electrical Engineering from Madurai Kamaraj University and joined ISRO in 2004. She has worked in Spacecraft Operations at Master Control Facility(MCF), Hassan and later moved to Indian Remote Sensing Satellite (IRS) Programme Management \& Systems Group (PMSG), UR Rao Satellite Centre. Her areas of interest include Project management, Intersatellite communication and Environment monitoring using Remote Sensing satellite data.

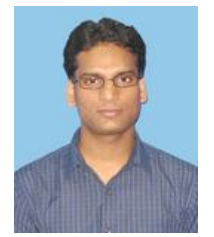

Vivek Kumar Gautam obtained B.Tech degree in Physical Sciences from Indian Institute of Space Science and Technology. He joined ISRO in 2011 and has worked in Indian Remote Sensing Satellite(IRS) Programme Management and Systems Group at UR Rao Satellite Centre. His areas of interest include Project management, System engineering, urban Remote Sensing and GIS applications.

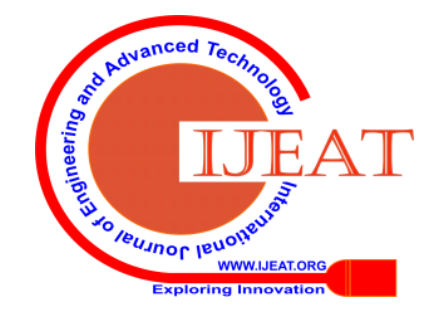

